\title{
Comparison of diagnostic accuracy among procalcitonin, C-reactive protein, and interleukin 6 for blood culture positivity in general ICU patients
}

\author{
Qin Wu, Hao Yang and Yan Kang ${ }^{*}$ (i)
}

Dear editor,

Despite various technological advances, it still usually takes at least 24 to $48 \mathrm{~h}$ to obtain a blood culture result. The subsequent delays in diagnosis and treatment of infection can negatively impact care in the intensive care unit (ICU). Biomarkers, such as procalcitonin (PCT), have been suggested as predictors of blood culture positivity in patients with different diseases $[1,2]$. However, the performances of PCT, C-reactive protein (CRP), and interleukin 6 (IL-6) in the prediction of blood culture positivity have never been assessed in a general ICU population.

We retrospectively assessed 13,377 consecutive adult ICU patients who did not have chronic kidney disease and who were discharged from a Chinese teaching hospital between January 1, 2016 and December 31, 2017. Among them, 534 patients who had suspected infection and whose PCT, CRP, and IL- 6 levels were measured when their blood was drawn for culture in accordance with standard procedure were analyzed. After the false-positive culture results as judged by two independent clinicians were excluded, the rate of positive blood culture was $7.49 \%$ (40 out of 534). Baseline characteristics between the blood culture-positive and blood culture-negative groups were comparable (Additional file 1). In contrast, the levels of PCT and IL-6 in the blood culture-positive group were significantly higher than those in the blood culture-negative group (Fig. 1). Receiver operating characteristic curves in Additional file 1 showed areas under the curve of 0.593 (95\% confidence interval (CI) $0.494-0.692)$ for PCT, $0.520(95 \%$ CI $0.400-0.693)$ for CRP, and 0.537 (95\% CI $0.457-0.617$ )

* Correspondence: kangyan@scu.edu.cn

Department of Critical Care Medicine, West China Hospital, Sichuan University, Chengdu, China

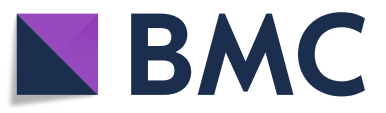

(c) The Author(s). 2018 Open Access This article is distributed under the terms of the Creative Commons Attribution 4.0 International License (http://creativecommons.org/licenses/by/4.0/), which permits unrestricted use, distribution, and

reproduction in any medium, provided you give appropriate credit to the original author(s) and the source, provide a link to the Creative Commons license, and indicate if changes were made. The Creative Commons Public Domain Dedication waiver (http://creativecommons.org/publicdomain/zero/1.0/) applies to the data made available in this article, unless otherwise stated. for IL-6. A logistic regression analysis revealed that levels of PCT of more than $2.435 \mathrm{ng} / \mathrm{mL}$ and IL- 6 of more than $264.95 \mathrm{ng} / \mathrm{mL}$ were independently associated with blood culture positivity. Moreover, when those two biomarkers were combined, values of PCT of more than $2.435 \mathrm{ng} / \mathrm{mL}$ and IL-6 of more than $264.95 \mathrm{ng} / \mathrm{mL}$ could result in a very high specificity (93.57\%) but low sensitivity (12.5\%).

Our results suggest that patients with suspected infection probably have positive blood culture results if both their PCT and IL-6 levels are high. Even with the limitation of a single-center experience, our results indicate that PCT, IL-6, and PCT plus IL-6 levels might be useful for helping physicians to rapidly identify patients who are at risk of bloodstream infection and to select an appropriate empirical therapy.

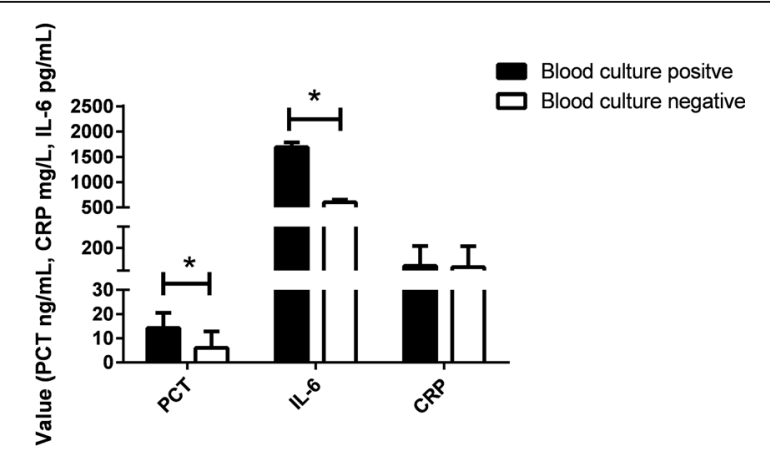

Fig. 1 Plot of procalcitonin (PCT), C-reactive protein (CRP), and interleukin 6 (IL-6) plasma concentrations in blood culture-positive and blood culture-negative groups. Significant differences (indicating as asterisk) in PCT and IL-6 were observed between groups 


\section{Additional file}

Additional file 1: Table S1. Demographics, clinical, and outcome data of patient cohort. (DOCX $30 \mathrm{~kb}$ )

\section{Abbreviations}

Cl: Confidence interval; CRP: C-reactive protein; ICU: Intensive care unit; IL6: Interleukin 6; PCT: Procalcitonin

\section{Acknowledgments}

Not applicable.

\section{Funding}

The work was supported by grants from the National Natural Science Foundation of China (81801892), the Wu Jieping Medical Foundation's special funding fund for clinical research (320.6750.17570), and the Sichuan University Postdoctoral Research Fund (2018SCU12036).

\section{Availability of data and materials}

Owing to health privacy concerns, the datasets generated and analyzed in this article are not publicly available. However, they are available from the corresponding author and will be obtainable by the public when construction of the database is complete.

\section{Authors' contributions}

QW and HY designed the whole study, conducted data analyses, and drafted the manuscript. YK supervised the whole project and performed data analysis. All authors read and approved the final manuscript.

\section{Ethics approval and consent to participate}

The clinical research ethics boards of the West China Hospital approved the study and waived the need for participants' informed consent because of the study's retrospective, anonymous, and non-interventional nature. All methods were performed in accordance with the relevant guidelines and regulations.

\section{Consent for publication}

Not applicable.

\section{Competing interests}

The authors declare that they have no competing interests.

\section{Publisher's Note}

Springer Nature remains neutral with regard to jurisdictional claims in published maps and institutional affiliations.

Received: 31 October 2018 Accepted: 21 November 2018

Published online: 17 December 2018

\section{References}

1. Naffaa M, Makhoul BF, Tobia A, Kaplan M, Aronson D, Azzam ZS, et al. Procalcitonin and interleukin 6 for predicting blood culture positivity in sepsis. Am J Emerg Med. 2014;32:448-51.

2. Oussalah A, Ferrand J, Filhine-Tresarrieu P, Aissa N, Aimone-Gastin I, Namour F, et al. Diagnostic Accuracy of Procalcitonin for Predicting Blood Culture Results in Patients With Suspected Bloodstream Infection: An Observational Study of 35,343 Consecutive Patients (A STROBE-Compliant Article). Medicine (Baltimore). 2015:94:e1774. 